\title{
Practice guideline recommendations summary: Treatment of tics in people with Tourette syndrome and chronic tic disorders
}

\author{
Tamara Pringsheim, MD, MSc, Michael S. Okun, MD, Kirsten Müller-Vahl, MD, Davide Martino, MD, PhD, \\ Joseph Jankovic, MD, Andrea E. Cavanna, MD, PhD, Douglas W. Woods, PhD, Michael Robinson, \\ Elizabeth Jarvie, MSW, LCSW, Veit Roessner, MD, Maryam Oskoui, MD, Yolanda Holler-Managan, MD, and \\ John Piacentini, PhD
}

Neurology ${ }^{\circledR}$ 2019;92:896-906. doi:10.1212/WNL.0000000000007466

\author{
Correspondence \\ American Academy of \\ Neurology \\ guidelines@aan.com
}

\begin{abstract}
\section{Objective}

To make recommendations on the assessment and management of tics in people with Tourette syndrome and chronic tic disorders.

\section{Methods}

A multidisciplinary panel consisting of 9 physicians, 2 psychologists, and 2 patient representatives developed practice recommendations, integrating findings from a systematic review and following an Institute of Medicine-compliant process to ensure transparency and patient engagement. Recommendations were supported by structured rationales, integrating evidence from the systematic review, related evidence, principles of care, and inferences from evidence.
\end{abstract}

\section{Results}

Forty-six recommendations were made regarding the assessment and management of tics in individuals with Tourette syndrome and chronic tic disorders. These include counseling recommendations on the natural history of tic disorders, psychoeducation for teachers and peers, assessment for comorbid disorders, and periodic reassessment of the need for ongoing therapy. Treatment options should be individualized, and the choice should be the result of a collaborative decision among patient, caregiver, and clinician, during which the benefits and harms of individual treatments as well as the presence of comorbid disorders are considered. Treatment options include watchful waiting, the Comprehensive Behavioral Intervention for Tics, and medication; recommendations are provided on how to offer and monitor these therapies. Recommendations on the assessment for and use of deep brain stimulation in adults with severe, treatment-refractory tics are provided as well as suggestions for future research.

\section{RELATED ARTICLE}

Comprehensive systematic review summary:

Treatment of tics in people with Tourette syndrome and chronic tic disorders Page 907

MORE ONLINE

ค Podcast

Dr. Jeffrey Ratliff talks with

Dr. Tamara Milka

Pringsheim about her practice guideline recommendations summary paper on the treatment of tics in people with Tourette syndrome and chronic tic disorders. NPub.org/q4j19p NEUROLOGY.

From the Department of Clinical Neurosciences, Psychiatry, Pediatrics and Community Health Sciences (T.P., D.M.), Cumming School of Medicine, University of Calgary, Alberta, Canada; Departments of Neurology and Neurosurgery (M.S.O.), Fixel Center for Neurological Diseases, University of Florida, Gainesville; Department of Psychiatry, Social Psychiatry, and Psychotherapy (K.M.-V.), Hannover Medical School, Germany; Department of Neurology (.J.), Baylor College of Medicine, Houston, TX; Department of Neuropsychiatry (A.E.C.), BSMHFT, University of Birmingham and Aston University, UK; Department of Psychology (D.W.W.), Marquette University, Milwaukee, Wl; Massachusetts Chapter (M.R.), Tourette Association of America, Bayside, NY; Waisman Center (E.J.), University Center for Excellence in Developmental Disabilities, University of Wisconsin, Madison; Technische Universitaet Dresden (V.R.), Germany; Departments of Pediatric and Neurology/Neurosurgery (M.O.), McGill University, Montréal, Canada; Department of Pediatrics (Neurology) (Y.H.-M.), Northwestern University Feinberg School of Medicine, Chicago, IL; and Department of Psychiatry and Biobehavioral Sciences (J.P.), Semel Institute for Neuroscience and Human Behavior, University of California Los Angeles.

Go to Neurology.org/N for full disclosures. Funding information and disclosures deemed relevant by the authors, if any, are provided at the end of the article.

Approved by the Guideline Development, Dissemination, and Implementation Subcommittee on July 29, 2017; by the AAN Practice Committee on October 15, 2018; and by the AAN Institute Board of Directors on February 12, 2019

This guideline was endorsed by the Child Neurology Society on September 4, 2018, and the European Academy of Neurology on September 5, 2018. 


\section{Glossary}

AAN = American Academy of Neurology; ADHD = attention-deficit/hyperactivity disorder; CBD = cannabidiol; CBIT = Comprehensive Behavioral Intervention for Tics; CBT = cognitive behavioral therapy; DBS = deep brain stimulation; DSM-5 = Diagnostic and Statistical Manual of Mental Disorders, 5th edition; EVID = evidence-based conclusions from the systematic review; HRT = habit reversal training; INFER = deductive inferences from other premises; OCD = obsessive-compulsive disorder; PRIN = generally accepted principles of care; RELA = strong evidence from related conditions; THC $=\delta$-9tetrahydrocannabinol; TS = Tourette syndrome; VMAT2 = vesicular monoamine transporter type 2.

This article presents the recommendations and suggestions for future research of an American Academy of Neurology (AAN) practice guideline on the treatment of tics in people with Tourette syndrome (TS) and chronic tic disorders. The complete and unabridged practice guideline is available as a data supplement at links.lww.com/WNL/A882. A companion article summarizes the systematic review findings and conclusions.

The AAN guideline committee convened a multidisciplinary panel to perform a systematic review and develop guideline recommendations, founded on the systematic review, for the assessment and treatment of tics. This article focuses on the guideline's recommendations and does not include detailed analysis of the systematic review. Readers are urged to review the unabridged guideline for a full assessment.

The systematic review synthesizes the evidence supporting the efficacy and harms of medical, behavioral, and neurostimulation treatments for tics. The treatment of tics must be individualized and based on collaborative decisions among patients, caregivers, and clinicians. Many people with tic disorders have psychiatric comorbidities, requiring clinicians to establish treatment priorities with their patients. While neurologists are often consulted to diagnose and treat tics, the identification and management of comorbid disorders is of prime importance and must be factored into management decisions. Therefore, while the level of obligation and associated verbs (must, should, may) state that treatments may or should be used, these recommendations pertain only to the situation in which the patient and clinician have determined that treatment is necessary and collaboratively discussed treatment options and priorities.

The authors of the practice guideline seek to make recommendations, based on evidence identified from the systematic review, general principles of care, and related evidence, regarding the following questions:

1. In children and adults with TS or a chronic tic disorder, when should clinicians and patients pursue treatment for tics?

2. In children and adults with TS or a chronic tic disorder who require treatment for tics, how should clinicians and

\section{$\oplus$ Supplemental Data}

Full measurement set

NPub.org/vsmv3e patients choose between evidence-based treatment options and determine the sequence or combinations of these treatments?

\section{Practice recommendations}

Much more than evidence must be considered when crafting practice recommendations. The evidence-based conclusions from our systematic review form the foundation of the AAN process, but other factors influence the structure of recommendations. The panel developed rationale statements that document, in a transparent manner, the deductive logic justifying each recommendation. These rationale statements precede each recommendation. Four types of premises can be used to support recommendations: (1) evidence-based conclusions from the systematic review (labeled EVID), (2) generally accepted principles of care (PRIN), (3) strong evidence from related conditions (RELA), and (4) deductive inferences from other premises (INFER). Recommendations must always be supported by at least one premise.

When there is sufficient evidence to support an inference for the use of an intervention (i.e., the balance of benefits and harms favors the intervention), the development panel assigns one of 3 recommendation designations: A, B, or C. Each designation corresponds to a helping verb that denotes the level of strength of the recommendation. Level A is the strongest recommendation level and is denoted by the use of the verb must. These recommendations are rare because they are based on high confidence in the evidence and require both a high magnitude of benefit and low risk. Level B corresponds to the verb should. Such recommendations tend to be more common because the requirements are less stringent but still based on the evidence and benefit-risk profile. Level C corresponds to the verb may. These recommendations represent the lowest allowable recommendation level that the AAN considers useful within the scope of clinical practice and accommodates the highest degree of practice variation.

Non-evidence-based factors that need to be transparently and systematically considered when formulating recommendations include (1) the relative value of the benefit compared with the risk, (2) the feasibility of complying with the intervention (e.g., the intervention's availability), (3) the cost of the intervention, and (4) the expected variation in patient preferences relative to the risks, burdens, and benefits of the intervention. The panel 
assigned levels of obligation (A, B, C, U, or R) to each recommendation by using a modified Delphi process that synthesizes all previously listed factors. The opinions of the guideline panel, with regard to the importance of each factor, were elicited through an online questionnaire, with statistical analysis of responses. The panel voted anonymously and independently on each recommendation in 3 rounds of online voting. Using precisely defined rules for consensus for each recommendation, the panel achieved consensus, revised the recommendation, or did not carry the recommendation forward. In some cases, the panel reviewed, revised, and revoted on recommendations on the basis of public commentary and other input during the guideline development process, reflecting the dynamic nature of this process. Suggestions for future research were created during guideline development.

\section{Counseling recommendation: Natural history of TS}

\section{Rationale}

Providing information to families about the natural history of a disorder can help inform treatment decisions (PRIN). Tics begin in childhood and demonstrate a waxing and waning course. Peak tic severity usually occurs between the ages of 10 and 12 years, with many children experiencing an improvement in tics in adolescence (RELA). ${ }^{1}$ A longitudinal study demonstrated that tic severity declined yearly during adolescence, with $18 \%$ of adolescents older than 16 years having no tics and $60 \%$ having minimal or mild tics 6 years after initial examination (RELA). ${ }^{2}$ There is no evidence that treatment is more effective the earlier it is started. As tics may improve with time, watchful waiting is an acceptable approach in individuals who do not experience any functional impairment from their tics (INFER). However, even in such cases, the Comprehensive Behavioral Intervention for Tics (CBIT) could be employed if the patient is motivated to attempt treatment (INFER). As a result of partial or complete remission during the natural course of the disorder, medication prescribed for tics in childhood may no longer be required over time (INFER).

\section{Recommendation 1a}

Clinicians must inform patients and their caregivers about the natural history of tic disorders (Level A).

\section{Recommendation $\mathbf{1 b}$}

Clinicians must evaluate functional impairment related to tics from the perspective of the patient and, if applicable, the caregiver (Level A).

\section{Recommendation 1c}

Clinicians should inform patients and caregivers that watchful waiting is an acceptable approach in people who do not experience functional impairment from their tics (Level B).

\section{Recommendation 1d}

Clinicians may prescribe CBIT as an initial treatment option relative to watchful waiting, for people with tics who do not experience functional impairment if they are motivated to attempt treatment (Level C).

\section{Recommendation 1e}

Physicians prescribing medications for tics must periodically re-evaluate the need for ongoing medical treatment (Level A).

\section{Psychoeducation, teacher and classroom}

\section{Rationale}

TS is common, affecting approximately $1 \%$ of schoolchildren (RELA). ${ }^{3}$ Psychoeducation about TS with peers can result in more positive attitudes toward a person with TS, while psychoeducation about TS with teachers can improve knowledge about the condition (RELA). ${ }^{4}$ Improving peers' attitudes about and teachers' knowledge of TS may positively affect people with TS (INFER).

\section{Recommendation 2}

Clinicians should refer people with TS to resources for psychoeducation for teachers and peers, such as the Tourette Association of America (Level B).

\section{Assessment and treatment of attention- deficit/hyperactivity disorder (ADHD) in children with tics}

\section{Rationale}

Comorbid ADHD is common in people with TS, with prevalence ranging from $30 \%$ to $50 \%$ (RELA). ${ }^{5,6}$ Several trials have specifically addressed the medical treatment of both ADHD and tics in children with both disorders. This includes trials of psychostimulants and atomoxetine, in which the aim was to demonstrate efficacy of these treatments for $\mathrm{ADHD}$ symptoms without concomitant worsening of tics. In children with tics and $\mathrm{ADHD}$, clonidine, clonidine plus methylphenidate, methylphenidate, and guanfacine are probably more likely than placebo to reduce tic severity (EVID) and reduce $\mathrm{ADHD}$ symptoms. In children with tics and $\mathrm{ADHD}$, atomoxetine does not worsen tics relative to placebo (EVID) and reduces $\mathrm{ADHD}$ symptoms. Comorbid $\mathrm{ADHD}$ is strongly associated with functional impairment in children with TS (RELA). ${ }^{7}$ While ADHD symptoms may improve in adolescence (RELA), ${ }^{2}$ adults with TS may require ongoing care for this comorbidity.

\section{Recommendation 3 a}

Clinicians should ensure an assessment for comorbid ADHD is performed in people with tics (Level B).

\section{Recommendation 3b}

Clinicians should evaluate the burden of ADHD symptoms in people with tics (Level B).

\section{Recommendation $3 c$}

In people with tics and functionally impairing $\mathrm{ADHD}$, clinicians should ensure appropriate $\mathrm{ADHD}$ treatment is provided (Level B). 
Assessment and treatment of obsessivecompulsive disorder (OCD) in children with tics

\section{Rationale}

Obsessive-compulsive behaviors are common in people with TS, with a comorbid diagnosis of OCD made in 10\%-50\% (RELA) ${ }^{5,6}$ Subanalyses of trials of interventions for OCD in children suggest that individuals with tics may not respond as well as those without tics to selective serotonin reuptake inhibitors but respond equally well to cognitive behavioral therapy (CBT) for OCD symptoms (RELA). ${ }^{8,9}$ For this reason, $\mathrm{CBT}$ is considered first-line treatment of $\mathrm{OCD}$ in individuals with tic disorders (INFER).

\section{Recommendation 4a}

Clinicians should ensure an assessment for comorbid OCD is performed in people with tics (Level B).

\section{Recommendation $\mathbf{4 b}$}

In people with tics and OCD, clinicians should ensure appropriate OCD treatment is provided (Level B).

\section{Other psychiatric comorbidities}

\section{Rationale}

Population-based and clinic-based studies have shown that people with TS are at high risk of other psychiatric comorbidities, including anxiety disorders, oppositional defiant disorder, and mood disorders (RELA). ${ }^{5,6}$ Comorbid mood disorders appear more prevalent in adolescents and adults than children and in those with greater tic severity (RELA). ${ }^{6,10}$ A matched case-cohort study using a national registry has shown an increased risk of dying by suicide and attempting suicide in people with TS compared with controls, which persisted after adjusting for psychiatric comorbidity. Persistence of tics beyond young adulthood, previous suicide attempts, and comorbid personality disorders increased the risk of death by suicide (RELA). ${ }^{11}$

\section{Recommendation 5a}

Clinicians must ensure appropriate screening for anxiety, mood, and disruptive behavior disorders is performed in people with tics (Level A).

\section{Recommendation 5b}

Clinicians must inquire about suicidal thoughts and suicide attempts in people with TS and refer to appropriate resources if present (Level A).

\section{Assessment of tic severity and treatment expectations}

\section{Rationale}

There are several rating scales available for measuring tic severity, with the Yale Global Tic Severity Scale the most extensively deployed and validated (RELA). ${ }^{12}$ Evaluation of the effect of treatment on tic severity in trials is measured using such scales (EVID). The use of validated scales to measure tic severity can aid the evaluation of treatment response in the clinical setting (INFER). While medications, behavioral therapy, and neurostimulation can result in meaningful reduction in tics (EVID), these interventions rarely result in complete cessation of tics.

\section{Recommendation 6a}

Clinicians may measure tic severity using a valid scale to assess treatment effects (Level C).

\section{Recommendation $\mathbf{6 b}$}

Clinicians must counsel patients that treatments for tics infrequently result in complete cessation of tics (Level A).

\section{Behavioral treatments}

\section{Rationale}

People with tics receiving CBIT are more likely than those receiving psychoeducation and supportive therapy to have reduced tic severity (EVID). CBIT is a manualized treatment program consisting of habit reversal training (HRT), relaxation training, and a functional intervention to address situations that sustain or worsen tics (RELA) ${ }^{13}$ The child and adult CBIT trials demonstrated the efficacy of an 8-session protocol, though cases complicated by poor tic awareness, treatment motivation, more severe tics, or substantial clinical comorbidity may benefit from a longer course of therapy. Most children (aged 9 years or older) and adults showing an initial positive response to CBIT will maintain their treatment gains for at least 6 months (EVID). CBIT can be effective for children younger than 9 years, though there is little evidence to determine efficacy in children of this age group (RELA). ${ }^{14}$ There is some evidence that the efficacy of CBIT for reducing tics is greater for patients not concurrently taking anti-tic medication (RELA). ${ }^{15}$ There is insufficient evidence to determine the relative efficacy of HRT compared with exposure and response prevention or educational group treatment in reducing tic severity (EVID). There is insufficient evidence to determine the relative efficacy of HRT by video conferencing compared with either face-to-face HRT or wait-list control for reducing tic severity (EVID). There is insufficient evidence to determine the efficacy of relaxation training for reducing tic severity (EVID). The evidence demonstrates no increased risk of adverse effects for people treated with CBIT compared with those treated with psychoeducation plus supportive therapy (EVID). The effect size for CBIT appears similar to effect sizes for medications (EVID). In light of clinician responsibility to optimally balance safety and effectiveness in treatment decisions (PRIN), CBIT should be considered as an initial treatment choice for tics (INFER). Given the effort required from patients or their families, along with its benign safety profile, CBIT is an acceptable intervention for people with tics that lead to psychosocial or physical impairment and who are motivated to participate in treatment (INFER).

\section{Recommendation 7a}

For people with tics who have access to CBIT, clinicians should prescribe CBIT as an initial treatment option relative to other psychosocial/behavioral interventions (Level B). 


\section{Recommendation $7 \mathrm{~b}$}

For people with tics who have access to CBIT, clinicians should offer CBIT as an initial treatment option relative to medication (Level B).

\section{Recommendation 7c}

Clinicians may prescribe CBIT delivered over teleconference or secure voice over Internet protocol delivery systems if face-toface options are unavailable in a patient care center. If CBIT is unavailable, other behavioral interventions for tics may be acceptable, such as exposure and response prevention (Level C).

\section{a-Agonists for the treatment of tics}

\section{Rationale}

People with tics receiving clonidine are probably more likely than those receiving placebo to have reduced tic severity, and people with tics receiving guanfacine are possibly more likely than those receiving placebo to have reduced tic severity, with the majority of trials conducted in children (EVID). In children with tics and comorbid $\mathrm{ADHD}$, clonidine and guanfacine have demonstrated beneficial effects on both tics and ADHD symptoms (EVID). The effect size of clonidine and guanfacine on tics appears larger in children with tics and ADHD compared with individuals with tics without a comorbid diagnosis of ADHD (EVID). Relative to placebo, clonidine is probably associated with higher rates of sedation, and guanfacine is probably associated with higher rates of drowsiness (EVID). A systematic review of $\alpha 2$ adrenergic agonists for ADHD in children and adolescents demonstrated hypotension, bradycardia, and sedation with both agents, and QTc prolongation with guanfacine extended release (RELA). ${ }^{16}$ Abrupt withdrawal of $\alpha 2$ adrenergic agonists may cause rebound hypertension (RELA). ${ }^{17}$

\section{Recommendation 8a}

Physicians should counsel individuals with tics and comorbid ADHD that $\alpha 2$ adrenergic agonists may provide benefit for both conditions (Level B).

\section{Recommendation $\mathbf{8 b}$}

Physicians should prescribe $\alpha 2$ adrenergic agonists for the treatment of tics when the benefits of treatment outweigh the risks (Level B).

\section{Recommendation 8c}

Physicians must counsel patients regarding common side effects of $\alpha 2$ adrenergic agonists, including sedation (Level A).

\section{Recommendation 8d}

Physicians must monitor heart rate and blood pressure in patients with tics treated with $\alpha 2$ adrenergic agonists (Level A).

\section{Recommendation $8 \mathrm{e}$}

Physicians prescribing guanfacine extended release must monitor the QTc interval in patients with a history of cardiac conditions, patients taking other QT-prolonging agents, or patients with a family history of long QT syndrome (Level A).

\section{Recommendation $8 \mathrm{f}$}

Physicians discontinuing a 2 adrenergic agonists must gradually taper them to avoid rebound hypertension (Level A).

\section{Antipsychotic treatment for tics}

\section{Rationale}

Haloperidol, risperidone, aripiprazole, and tiapride are probably more likely than placebo to reduce tic severity (EVID), and pimozide, ziprasidone, and metoclopramide are possibly more likely than placebo to reduce tic severity (EVID). There is insufficient evidence to determine the relative efficacy of these drugs (EVID). Relative to placebo, the evidence demonstrates a higher risk of drug-induced movement disorders with haloperidol, pimozide, and risperidone (EVID), a higher risk of weight gain with risperidone and aripiprazole (EVID), a higher risk of somnolence with risperidone, aripiprazole, and tiapride (EVID), a higher risk of $\mathrm{QT}$ prolongation with pimozide (EVID), and a higher risk of elevated prolactin with haloperidol, pimozide, and metoclopramide (EVID). Systematic reviews of trials and cohort studies demonstrate a higher risk of drug-induced movement disorders (including tardive dyskinesia, drug-induced parkinsonism, akathisia, acute dystonia, and tardive dystonia), weight gain, adverse metabolic side effects, prolactin increase, and QT prolongation with both firstand second-generation antipsychotics across psychiatric and neurologic conditions (RELA). ${ }^{18,19}$ The long-term use of metoclopramide is associated with tardive dyskinesia, resulting in a black box warning from the US Food and Drug Administration. ${ }^{20}$ The relative propensity for these adverse effects varies by agent and is often dose-dependent (RELA). Physicians have a duty to monitor the effectiveness and safety of prescribed medications (PRIN), and evidence-based monitoring protocols are available. ${ }^{21}$ Abrupt discontinuation of antipsychotic medications can cause withdrawal dyskinesias (RELA). ${ }^{22,23}$

\section{Recommendation 9a}

Physicians may prescribe antipsychotics for the treatment of tics when the benefits of treatment outweigh the risks (Level C).

\section{Recommendation 9b}

Physicians must counsel patients on the relative propensity of antipsychotics for extrapyramidal, hormonal, and metabolic adverse effects to inform decision-making on which antipsychotic should be prescribed (Level A).

\section{Recommendation 9c}

Physicians prescribing antipsychotics for tics must prescribe the lowest effective dose to decrease the risk of adverse effects (Level A).

\section{Recommendation 9d}

Physicians prescribing antipsychotics for tics should monitor for drug-induced movement disorders and for metabolic and hormonal adverse effects of antipsychotics, using evidencebased monitoring protocols (Level B). 


\section{Recommendation 9e}

Physicians prescribing antipsychotics for tics must perform electrocardiography and measure the $\mathrm{QT}_{\mathrm{c}}$ interval before and after starting pimozide or ziprasidone, or if antipsychotics are coadministered with other drugs that can prolong the QT interval (Level A).

\section{Recommendation $9 f$}

When attempting to discontinue antipsychotics for tics, physicians should gradually taper medications over weeks to months to avoid withdrawal dyskinesias (Level B).

\section{Botulinum toxin injections for tics}

Botulinum toxin injections with onabotulinumtoxinA are probably more likely than placebo to reduce tic severity in adolescents and adults (EVID). Premonitory urges may also be improved by botulinum toxin injections (RELA). ${ }^{24}$ Relative to placebo, onabotulinumtoxinA is associated with higher rates of weakness (EVID). Hypophonia is a common side effect of botulinum toxin injections in the laryngeal muscles for vocal tics (RELA). ${ }^{25}$ The effects of botulinum toxin injections last 12-16 weeks, after which treatment needs to be repeated (PRIN).

\section{Recommendation 10a}

Physicians may prescribe botulinum toxin injections for the treatment of adolescents and adults with localized and bothersome simple motor tics when the benefits of treatment outweigh the risks (Level C).

\section{Recommendation 10b}

Physicians may prescribe botulinum toxin injections for the treatment of older adolescents and adults with severely disabling or aggressive vocal tics when the benefits of treatment outweigh the risks (Level C).

\section{Recommendation 10c}

Physicians must counsel individuals with tics that botulinum toxin injections may cause weakness and hypophonia, and that all effects are temporary (Level A).

\section{Topiramate for the treatment of tics}

\section{Rationale}

Topiramate is possibly more likely than placebo to reduce tic severity (EVID). In patients with mild but troublesome tics who are not obtaining a satisfactory response or experience adverse effects from other treatments, topiramate may be a useful alternative. While generally well-tolerated at low doses (25-150 mg/day) it may cause adverse effects, including cognitive and language problems, somnolence, and weight loss, and may increase the risk of renal stones (RELA). ${ }^{26-28}$

\section{Recommendation 11a}

Physicians should prescribe topiramate for the treatment of tics when the benefits of treatment outweigh the risks (Level B).

\section{Recommendation 11b}

Physicians must counsel patients regarding common adverse effects of topiramate, including cognitive and language problems, somnolence, weight loss, and an increased risk of renal stones (Level A).

\section{Cannabis-based medications for the treatment of patients with TS}

\section{Rationale}

Some patients with TS use cannabis as a self-medication for tics and comorbidities (RELA). ${ }^{29}$ There is limited evidence that $\delta$-9-tetrahydrocannabinol (THC), dronabinol, is possibly more likely than placebo to reduce tic severity in adults with TS (EVID). There is insufficient evidence to determine whether the efficacy of nabiximols, nabilone, and cannabidiol (CBD), as well as different strains of medicinal cannabisstandardized for different levels of THC and CBD—is similar to THC. Compared with placebo, cannabis-based medications are associated with increased risk of short-term adverse events, most commonly dizziness, dry mouth, and fatigue (RELA). ${ }^{30}$ There is no evidence that controlled treatment with cannabis-based medication may induce addiction to cannabinoids. Acute withdrawal of cannabinoids is generally safe and well-tolerated without significant adverse events (RELA). ${ }^{30,31}$ Cannabis-based medications should be avoided in children and adolescents, not only due to a paucity of evidence but due to the association between cannabis exposure in adolescence and potentially harmful cognitive and affective outcomes in adulthood (RELA, PRIN)..$^{32}$ Cannabisbased medication should not be used in women who are pregnant or breastfeeding or in patients with psychosis (PRIN). Prescription of and access to medical marijuana varies by region; practitioners must abide by regional legislation on the use of medical marijuana (PRIN).

\section{Recommendation 12a}

Due to the risks associated with cannabis use and widespread self-medication with cannabis for tics, where regional legislation and resources allow, physicians must offer to direct patients to appropriate medical supervision when cannabis is used as self-medication for tics (Level A). Appropriate medical supervision would entail education and monitoring for efficacy and adverse effects.

\section{Recommendation 12b}

Where regional legislation allows, physicians may consider treatment with cannabis-based medication in otherwise treatment-resistant adults with clinically relevant tics (Level C).

\section{Recommendation 12c}

Where regional legislation allows, physicians may consider treatment with cannabis-based medication in adults with TS who already use cannabis efficiently as a self-medication in order to better control and improve quality of treatment (Level C).

\section{Recommendation 12d}

Where regional legislation allows, physicians prescribing cannabis-based medication must prescribe the lowest effective dose to decrease the risk of adverse effects (Level A). 


\section{Recommendation $12 \mathrm{e}$}

Physicians prescribing cannabis-based medication must inform patients that medication may impair driving ability (Level A).

\section{Recommendation $12 \mathrm{f}$}

Physicians prescribing cannabis-based medication to patients with TS must periodically reevaluate the need for ongoing treatment (Level A).

\section{Deep brain stimulation (DBS) for tics in the setting of TS}

\section{Rationale}

Patients with severe TS, resistant to medical and behavioral therapy, may benefit from the application of DBS. An important challenge and limitation in the evaluation of the evidence around DBS in TS is that, even in expert DBS centers, few operations per year are performed. Furthermore, there is limited information from randomized clinical trials for analysis and interpretation. There is no consensus on the optimal brain target for the treatment of tics, but the following regions have been stimulated in patients with TS: the centromedian thalamus, the globus pallidus internus (ventral and dorsal), the globus pallidus externus, the subthalamic nucleus, and the ventral striatum/ ventral capsular nucleus accumbens region. DBS of the anteromedial globus pallidus is probably more likely than sham stimulation to reduce tic severity (EVID). There is insufficient evidence to determine the efficacy of DBS of the thalamus or the centromedian-parafascicular complex region of the thalamus in reducing tic severity (EVID). Complications of treatment, including infection and removal of hardware, appear more common with TS (EVID) than with other neurologic conditions.

Recommendations from the Movement Disorders Society suggest that, when DBS is used in TS, best practices used for other DBS applications are followed, including confirmation of diagnosis, use of multidisciplinary screening, and stabilization of psychiatric comorbidities inclusive of active suicidality (RELA). ${ }^{33}$ Appropriate patient selection is one of the most important predictors of success of DBS treatment, making multidisciplinary evaluation essential (RELA). ${ }^{34} \mathrm{Be}$ cause of the complexity of the patient population, centers performing DBS have been encouraged to screen candidates preoperatively and to follow them postoperatively. There has been concern about high risk of suicide and other negative psychiatric sequelae in patients with TS not screened and monitored for depression, anxiety, and bipolar tendencies. The largest available randomized trials of DBS have revealed benefits on motor and phonic tics for the ventral globus pallidus internus and the centromedian thalamic region target; however, these studies have raised methodologic concerns that need to be addressed in future trials (RELA). ${ }^{35}$ There is little information on the effects of DBS on psychiatric comorbidities and on the efficacy of DBS in children with TS.

\section{Recommendation 13a}

Physicians must use a multidisciplinary evaluation (psychiatrist or neurologist, neurosurgeon, and neuropsychologist) to establish when the benefits of treatment outweigh the risks of prescribing DBS for medication-resistant motor and phonic tics (Level A).

\section{Recommendation 13b}

Physicians should confirm the DSM-5 diagnosis of TS and exclude secondary and functional tic-like movements when considering DBS for medication-resistant tics (Level B).

\section{Recommendation 13c}

A mental health professional must screen patients preoperatively and follow patients postoperatively for psychiatric disorders that may impede the long-term success of the therapy (Level A).

\section{Recommendation 13d}

Physicians must confirm that multiple classes of medication (antipsychotics, dopamine depleters, $\alpha 2$ agonists) and behavioral therapy have been administered (or are contraindicated) before prescribing DBS for tics (Level A).

\section{Recommendation $13 \mathrm{e}$}

Physicians may consider DBS for severe, self-injurious tics, such as severe cervical tics that result in spinal injury (Level C).

\section{Suggestions for future research}

1. Future research on behavioral interventions for tics should include comparisons of the relative efficacy of CBIT vs pharmacotherapy. Additional research should be conducted on treatment sequencing and decisionmaking and for whom particular sequences of treatment are most effective. Further research should continue to test the efficacy of other behavioral treatments, including exposure and response prevention, mindfulness-based treatments, or more global tic-related interventions. ${ }^{36}$ As the evidence is insufficient at present to conclude that CBIT delivered by teleconference is as effective as faceto-face treatment, further well-designed studies with adequate sample sizes are needed to establish noninferiority. Additional work to more accurately characterize the neurocognitive and behavioral mechanism of action underlying CBIT will be necessary to enhance the overall effectiveness and inform patient-treatment matching algorithms. ${ }^{37}$

2. Future research on medications for tics should include noninferiority trials of agents commonly used for the treatment of tics for which limited evidence from randomized controlled trials is available. Agents for which evidence is promising but limited include the first-generation antipsychotic fluphenazine. ${ }^{38-40}$ Trials are currently underway with the selective $\mathrm{D} 1$ antagonist ecopipam and evidence on the efficacy of this drug is expected soon.

3. The dopamine depleters, tetrabenazine, deutetrabenazine, and valbenazine, act by blocking vesicular monoamine transporter type 2 (VMAT2). Although no randomized controlled trials have been published with the VMAT2 
inhibitors in the treatment of tics, these drugs are increasingly used off-label. When appropriately dosed, these drugs are generally well-tolerated but may be associated with drowsiness, depression, and parkinsonism. Although an initial phase II trial of valbenazine did not reach the primary endpoint in adults and children with TS, this was thought to be due to underdosing. Further and better-designed trials are currently underway with valbenazine and deutetrabenazine for the treatment of tics. ${ }^{41-43}$

4. Our systematic review included 3 different traditional Chinese medicine products: the 5-Ling granule, ${ }^{44}$ the Ningdong granule formulated by Zhao, ${ }^{45}$ and the Ningdong granule formulated by Wang. ${ }^{46}$ We did not make formal recommendations for or against the use of these compounds, all of which reported superiority over placebo. Our panel had concerns about the inclusion criteria for the 5-Ling granule study, as participants also had a condition fitting the "excessive subtype" in traditional Chinese medicine-based diagnosis (see full and unabridged practice guideline for full details), with no equivalent diagnosis in Western medicine or clear understanding of pathophysiology. Furthermore, this study excluded children with the 2 most common comorbidities seen with TS: ADHD and OCD. There are therefore concerns with respect to the generalizability of these findings. Finally, the availability of these compounds outside of the trials is unknown and safety concerns remain regarding the ingredients-the Ningdong granule formulated by Wang contains human dried placenta. Further research and information on the safety and reliability of production of these agents is required before formal recommendations can be made.

5. There is a need for more long-term studies of drug efficacy and adverse effects as well as the efficacy and safety of medication combinations for severe tics resistant to monotherapy.

6. Few studies have been performed investigating the efficacy and safety of cannabis-based medicine in children with various diseases. Recently it was reported that $\mathrm{CBD}$ may significantly reduce convulsive seizure frequency in children with Dravet syndrome. ${ }^{47}$ There is preliminary evidence that THC might also be effective in children for vomiting due to antineoplastic treatment ${ }^{48,49}$ and in treatment-resistant spasticity. ${ }^{50}$ There is increasing evidence that cannabis-based medicine might be effective in adults with $\mathrm{TS}^{51}{ }^{51} \mathrm{~A}$ recent press release for a singledose study of a first-in-class small molecule inhibitor of monoacylglycerol lipase, ABX-1431, which regulates one of the key natural activators of the central cannabinoid $\mathrm{CB} 1$ receptor, suggests efficacy for the treatment of tics. ${ }^{52}$

7. Case reports and case series have comprised the majority of the outcomes data on the efficacy of DBS for TS. An international DBS registry ${ }^{53}$ has been developed to collect data on DBS outcomes in patients with TS implanted in various centers. The registry also collects information about response to nonstandardized selection criteria, various brain targets, differences in hardware, and variability in the programming parameters used. The goal of future research on DBS in TS should be to improve outcomes and quality of life by conducting well-designed multicenter studies, share data across centers, uncover best practices, and provide critical information to regulatory agencies that will lead to approval of DBS in TS. There are important limitations to the currently available trials using DBS in TS. The uncertainty in optimal target and the individual variability in programming and management between participants make trials challenging. Recent research on DBS in TS has revealed the intriguing possibility that it may not be necessary to have the devices activated continuously as has been the standard for other movement disorders. Moreover, adaptive closed-loop DBS is being explored in an ongoing clinical trial.

8. Future research on the effect of special diets, nutritional supplements, and exercise on tic severity is needed.

\section{Author contributions}

Dr. Pringsheim: study concept and design, acquisition of data, analysis or interpretation of data, drafting/revising the manuscript, critical revision of the manuscript for important intellectual content, study supervision. Dr. Okun: study concept and design, critical revision of the manuscript for important intellectual content. Dr. Müller-Vahl: study concept and design, critical revision of the manuscript for important intellectual content. Dr. Martino: study concept and design, critical revision of the manuscript for important intellectual content. Dr. Jankovic: study concept and design, critical revision of the manuscript for important intellectual content. Dr. Cavanna: study concept and design, critical revision of the manuscript for important intellectual content. Dr. Woods: study concept and design, critical revision of the manuscript for important intellectual content. M. Robinson: study concept and design, critical revision of the manuscript for important intellectual content. E. Jarvie: study concept and design, critical revision of the manuscript for important intellectual content. Dr. Roessner: study concept and design, critical revision of the manuscript for important intellectual content. Dr. Oskoui: study concept and design, acquisition of data, analysis or interpretation of data, critical revision of the manuscript for important intellectual content. Dr. HollerManagan: study concept and design, acquisition of data, analysis or interpretation of data, critical revision of the manuscript for important intellectual content. Dr. Piacentini: study concept and design, critical revision of the manuscript for important intellectual content.

\section{Study funding}

This practice guideline was developed with financial support from the American Academy of Neurology (AAN). Authors who serve as AAN subcommittee members (Y.H.-M., M.O.) or as methodologists (T.P.), or who are AAN staff members 
(S.M.), were reimbursed by the AAN for expenses related to travel to subcommittee meetings where drafts of manuscripts were reviewed.

\section{Disclosure}

T. Pringsheim has no disclosures to report. M. Okun has declared nonfinancial support from the Parkinson's Foundation (PF) as National Medical Director; has received grants from the NIH, PF, Michael J. Fox Foundation (MJFF), and the Tourette Association of America (TAA); serves on the TAA Medical Advisory Board; is a member of the Board of Directors of Movements Disorders, Tremor and Hyperkinetic Disorders; has received royalties from publishing on Amazon, Smashwords, Taylor, Demos, and Books4Patients; has received continuing medical education speaker fees from Medscape/Web MD, Mededicus, PeerView, the AAN, and the Movement Disorders Society; provides clinical care for patients with Tourette syndrome; has received financial or material research support or compensation from the NIH, the $\mathrm{PF}$, the MJFF, and the TAA; and has given expert testimony on medicolegal cases (approximately 10 years ago) but had no court appearances. K. Müller-Vahl has nonfinancial competing interests as a member of the TAA medical advisory board, the scientific advisory board of the German Tourette Association (TGD), the board of directors of the German (ACM) and the International (IACM) Association for Cannabinoid Medicines, and the committee of experts for narcotic drugs at the federal opium bureau of the Federal Institute for Drugs and Medical Devices (BfArM) in Germany; has received consultant's honoraria from Abide Therapeutics, Fundacion Canna, and Therapix Biosiences, and speaker's fees from Tilray, and is a consultant for Zynerba Pharmaceuticals; has served as a guest editor for Frontiers in Neurology on the research topic "The neurobiology and genetics of Gilles de la Tourette syndrome: new avenues through large-scale collaborative projects" and is an associate editor for Cannabis and Cannabinoid Research; has performed several clinical studies related to Tourette syndrome, including randomized controlled trials using cannabinoids and behavioral therapy; has received financial or material research support from the German Ministry of Education and Research (BMBF), German Research Society (Deutsche Forschungsgemeinschaft $[D F G])$, European Union, Tourette Gesellschaft Deutschland e.V., Else-Kroner-Fresenius-Stiftung, and GW, Almirall, Abide Therapeutics, and Therapix Biosiences; and has received royalties from Medizinisch Wissenschaftliche Verlagsgesellschaft Berlin. D. Martino has no disclosures to report. J. Jankovic has served on advisory boards of, and received reimbursement for travel expenses from, Adamas Pharmaceuticals, Inc., Allergen, Inc., and Teva Pharmaceuticals Industries Ltd.; serves as a journal editor, an associate editor, or as a member of an editorial advisory board for Parkinson and Related Disorders, Acta Neurologica Scandinavica Journal of the Neurological Sciences, Medlink, Neurotherapeutics, and Tremor and Other Hyperkinetic Movements; has received royalties from publishing with Cambridge, Elsevier, Future Science Group, Hodder Arnold, Lippincott Williams \&
Wilkins, and Wiley-Blackwell; has received honoraria from Adamas Pharmaceuticals, Inc. and Teva Pharmaceuticals Industries Ltd.; has given botulinum neurotoxin injections; and has received research grants from Adamas Pharmaceutical, Inc. and Allergan, Inc. A. Cavanna has had nonfinancial competing interests at the Royal College of Psychiatrists, Faculty of Neuropsychiatry, Movement Disorders; has received funding for travel from the TAA; has served as a journal editor, an associate editor, or an editorial advisory board member for Behavioral Neurology and Epilepsy and Behavior; has received royalties from Oxford University Press; and has received personal compensation from speakers' bureaus with UCB Pharma, Eisai, and Janssen-Cilag. D. Woods has a nonfinancial competing interest as a member of the TAA Medical Advisory Board; has received royalties from Guilford Press, Oxford University Press, and Springer Press; and has received honoraria from speaking from the TAA. M. Robinson has a nonfinancial competing interest in serving as co-Chair for the Massachusetts State Chapter of the Tourette Association of America Board of Directors. E. Jarvie has declared a nonfinancial competing interest in serving as member of the Wisconsin Tourette Syndrome Association Board of Directors. V. Roessner serves on an advisory board for the German Tourette Society and the German Society of ObsessiveCompulsive Disorder; has received funding for travel from Actelion, Lilly, MEDICE, Novartis, and Shire; serves a journal editor, associate editor, or member of an advisory board for European Child and Adolescent Psychiatry, Zeitschrift fur Kinder-und Jugendpsychiatrie, Neuropsychiatrie, Behavioral Neurology, and Scientific Reports; has received honoraria from Actelion, Lilly, MEDICE, Novartis, and Shire; has received financial or material research support or compensation from the government entities of the European Union, DFG, BMBF, and KSV Sachsen; and has received support from academic entities such as Tourette Gesellschaft Deutschland e.V., Roland-Ernst-Stiftung, Friede-Springer-Stiftung, and Else-Kroner-Fresenius-Stiftung, and from commercial entities such as Novartis. M. Oskoui has received funding for travel from the AAN; has received research support from the government entities of Fonds de Recherche Sante du Québec, Canada Institute of Health Research, McGill University Research Institute, the SickKids Foundation, Cerebral Palsy Alliance Foundation, and Kids Brain Health Network for research in cerebral palsy; serves on the data safety monitoring board for Avexis; has received financial compensation for consulting work for Biogen and Roche; and has received research support as site PI for Ionis, Biogen, Roche, and Cytokinetics for clinical trials in spinal muscular atrophy. Y. Holler-Managan has received funding for travel to the AAN and has served as member of an editorial advisory board for Neurology Now. J. Piacentini has received funding for travel from the TLC Foundation for Body-Focused Repetitive Behaviors (BFRBs); has received travel funding and speaking honoraria from the TAA, the International ObsessiveCompulsive Disorder Foundation (IOCDF), the Karolinska Institutet, University of Modena, OCD New Jersey, New York University, the Child Mind Institute, the University of 
Southern Maine, Florida International University, and the Spanish Association for Child and Adolescent Psychiatry; has received royalties from Guilford Press and Oxford University Press; has performed behavior therapy for tics approximately $50 \%$ of his clinical time; and has received financial or material support from Pfizer Pharmaceuticals, the National Institute of Mental Health of the NIH, and from the academic entity of 501C3s, the TAA, TLC Foundation for BFRBs, and the Pettit Family Foundation. Go to Neurology.org/N for full disclosures.

\section{Disclaimer}

Practice guidelines, practice advisories, comprehensive systematic reviews, focused systematic reviews, and other guidance published by the AAN and its affiliates are assessments of current scientific and clinical information provided as an educational service. The information (1) should not be considered inclusive of all proper treatments, methods of care, or as a statement of the standard of care; (2) is not continually updated and may not reflect the most recent evidence (new evidence may emerge between the time information is developed and when it is published or read); (3) addresses only the question(s) specifically identified; (4) does not mandate any particular course of medical care; and (5) is not intended to substitute for the independent professional judgment of the treating provider, as the information does not account for individual variation among patients. In all cases, the selected course of action should be considered by the treating provider in the context of treating the individual patient. Use of the information is voluntary. AAN provides this information on an "as is" basis, and makes no warranty, expressed or implied, regarding the information. AAN specifically disclaims any warranties of merchantability or fitness for a particular use or purpose. AAN assumes no responsibility for any injury or damage to persons or property arising out of or related to any use of this information or for any errors or omissions.

\section{Conflict of interest}

The AAN is committed to producing independent, critical, and truthful clinical practice guidelines (CPGs). Significant efforts are made to minimize the potential for conflicts of interest to influence the recommendations of this CPG. To the extent possible, the AAN keeps separate those who have a financial stake in the success or failure of the products appraised in the CPGs and the developers of the guidelines. Conflict of interest forms were obtained from all authors and reviewed by an oversight committee prior to project initiation. AAN limits the participation of authors with substantial conflicts of interest. The AAN forbids commercial participation in, or funding of, guideline projects. Drafts of the guideline have been reviewed by at least 3 AAN committees, a network of neurologists, Neurology peer reviewers, and representatives from related fields. The AAN Guideline Author Conflict of Interest Policy can be viewed at www.aan.com. For complete information on this process, access the 2011 AAN process manual, as amended. ${ }^{54}$

\section{Publication history}

Received by Neurology June 15, 2018. Accepted in final form November 21, 2018.

\section{References}

1. Bloch MH, Peterson BS, Scahill L, et al. Adulthood outcome of tic and obsessive compulsive symptom severity in children with Tourette syndrome. Arch Pediatr Adolesc Med 2006;160:65-69.

2. Groth C, Mol Debes N, Rask CU, Lange T, Skov L. Course of Tourette syndrome and comorbidities in a large prospective clinical study. J Am Acad Child Adolesc Psychiatry $2017 ; 56: 304-312$.

3. Knight T, Steeves T, Day L, Lowerison M, Jette N, Pringsheim T. Prevalence of tic disorders: a systematic review and meta-analysis. Pediatr Neurol 2012;47: 77-90.

4. Nussey C, Pistrang N, Murphy T. How does psychoeducation help? A review of the effects of providing information about Tourette syndrome and attention-deficit/ hyperactivity disorder. Child Care Health Dev 2013;39:617-627.

5. Kurlan R, Como PG, Miller B, et al. The behavioural spectrum of tic disorders: a community based study. Neurology 2002;59:414-420.

6. Hirschtritt ME, Lee PC, Pauls DL, et al. Lifetime prevalence, age of risk, and genetic relationships of comorbid psychiatric disorders in Tourette syndrome. JAMA Psychiatry $2015 ; 72: 325-333$.

7. Sukhodolsky DG, Scahill L, Zhang H, et al. Disruptive behaviour in children with Tourette's syndrome: association with ADHD comorbidity, tic severity, and functional impairment. J Am Acad Child Adolesc Psychiatry 2003;42:98-105.

8. March JS, Franklin ME, Leonard H, et al. Tics moderate treatment outcome with sertraline but not cognitive-behavior therapy in pediatric obsessive-compulsive disorder. Biol Psychiatry 2007;61:344-347.

9. Conelea CA, Walther MR, Freeman JB, et al. Tic-related obsessive-compulsive disorder (OCD): phenomenology and treatment outcome in the Pediatric OCD Treatment Study II. J Am Acad Child Adolesc Psychiatry 2014;53:1308-1316.

10. Chou I, Lin H, Lin C, Sung F, Kao C. Tourette syndrome and risk of depression: a population-based cohort study in Taiwan. J Dev Behav Pediatr 2013;34:181-185.

11. Fernandez de la Cruz L, Rydell M, Runeson B, et al. Suicide in Tourette's and chronic tic disorders. Biol Psychiatry 2017;82:111-118.

12. Martino D, Pringsheim T, Cavanna AE, et al. Systematic review of severity scales and screening instruments for tics: critique and recommendations. Mov Disord 2017;32: 467-473.

13. Woods DW, Piacentini J, Chang S, et al. Managing Tourette Syndrome: A Behavioral Intervention for Children and Adults Therapist Guide. New York: Oxford University Press; 2008.

14. Woods DW, Miltenberger RG. A review of habit reversal with childhood habit disorders. Educ Treat Child 1996;19.

15. Sukhodolsky DG, Woods DW, Piacentini J, et al. Moderators and predictors of response to behaviour therapy for tics in children and adults with Tourette's disorder and chronic tic disorders. Neurology 2017;88:1-8.

16. Hirota T, Schwartz S, Correll CU. Alpha-2 agonists for attention-deficit/hyperactivity disorder in youth: a systematic review and meta-analysis of monotherapy and add-on trials to stimulant therapy. J Am Acad Child Adolesc Psychiatry 2014;53:153-173.

17. Reid JL, Campbell BC, Hamilton CA. Withdrawal reactions following cessation of central alpha-adrenergic receptor agonists. Hypertension 1984;6:71-75.

18. Pringsheim T, Lam D, Ching H, Patten S. Metabolic and neurological complications of second generation antipsychotic use in children: a systematic review and metaanalysis of randomized controlled trials. Drug Saf 2011;34:651-668.

19. Leucht S, Cipriani A, Spineli L, et al. Comparative efficacy and tolerability of 15 antipsychotic drugs in schizophrenia: a multiple-treatments meta-analysis. Lancet 2013;382:951-962.

20. Alaven. Reglan Product Monograph. Stockholm; Alaven: 2010.

21. Pringsheim T, Panagiotopoulos C, Davidson J, Ho J. Evidence-based recommendations for monitoring safety of second generation antipsychotics in children and youth. Paediatr Child Health 2011;16:581-589.

22. Campbell M, Armenteros JL, Malone RP, Adams PB, Eisenberg ZW, Overall JE. Neuroleptic-related dyskinesias in autistic children: a prospective, longitudinal study. J Am Acad Child Adolesc Psychiatry 1997;36:835-843.

23. Kumra S, Jacobsen LK, Lenane M, et al. Case series: spectrum of neuroleptic-induced movement disorders and extrapyramidal side effects in childhood-onset schizophrenia. J Am Acad Child Adolesc Psychiatry 1997;37:221-227.

24. Rath JJ, Tavy DL, Wertenbroek AA, van Woerkom TC, de Bruijn SF. Botulinum toxin type A in simple motor tics: short-term and long-term treatment-effects. Parkinsonism Relat Disord 2010;16:478-481.

25. Porta M, Maggioni G, Ottaviani F, Schindler A. Treatment of phonic tics in patients with Tourette's syndrome using botulinum toxin type A. Neurol Sci 2003;24:420-423.

26. Donegan S, Dixon P, Hemming K, Tudur-Smith C, Marson A. A systematic review of placebo-controlled trials of topiramate: how useful is a multiple-indications review for evaluating the adverse events of an antiepileptic drug? Epilepsia 2015;56:1910-1920.

27. Dell'Orto VG, Belotti EA, Goeggel-Simonetti B, et al. Metabolic disturbances and renal stone promotion on treatment with topiramate: a systematic review. $\mathrm{Br} \mathrm{J}$ Clin Pharmacol 2014;77:958-964.

28. Kramer CK, Leitao CB, Pinto LC, Canani LH, Azevedo MJ, Gross JL. Efficacy and safety of topiramate on weight loss: a meta-analysis of randomized controlled trials. Obes Rev 2011;12:e338-347. 
29. Hazenkamp A, Ware MA, Mueller-Vahl K, Abrams D, Grotenhermen F. The medicinal use of cannabis and cannabinoids: an international cross-sectional survey on administration forms. J Psychoactive Drugs 2013;45:199-210.

30. Whiting PF, Wolff RF, Deshpande S, Di Nisio M, Duffy S, Hernandez AV. Cannabinoids for medical use: a systematic review and meta-analysis. JAMA 2015;313:2456-2473.

31. Robson P. Abuse potential and psychoactive effects of delta-9-tetrahydrocannabinol and cannabidiol oromucosal spray (Sativex), a new cannabinoid medicine. Expert Opin Drug Saf 2011;10:675-685.

32. Levine A, Clemenza K, Rynn M, Lieberman J. Evidence for the risks and consequences of adolescent cannabis exposure. J Child Adolesc Psychopharmacol 2017; 56:214-225.

33. Schrock LE, Mink J, Woods DW, et al. Tourette syndrome deep brain stimulation: a review and updated recommendations. Mov Disord 2015;30:448-471.

34. Okun MS, Tagliati M, Pourfar M, et al. Management of referred deep brain stimulation failures: a retrospective analysis from two movement disorders centers. Arch Neurol 2005;62:1250-1255.

35. Jimenez-Shahed J. Design challenges for stimulation trials of Tourette's syndrome. Lancet Neurol 2015;14:563-565.

36. McGuire JF, Arnold E, Park JM, et al. Living with tics: reduced impairment and improved quality of life for youth with chronic tic disorders. Psychiatry Res 2015;225: 571-579.

37. McGuire JF, Piacentini J, Scahill L, et al. Bothersome tics in patients with chronic tic disorders: characteristics and individualized treatment response to behaviour therapy. Behav Res Ther 2015;70:56-63.

38. Borison RL, Ang L, Hamilton WJ, Diamond BI, Davis JM. Treatment approaches in Gilles de la Tourette syndrome. Brain Res Bull 1983;11:205-208.

39. Goetz CG, Tanner CM, Klawans HL. Fluphenazine and multifocal tic disorders. Arch Neurol 1984;41:271-272.

40. Wijemanne S, Wu LJ, Jankovic J. Long-term efficacy and safety of fluphenazine in patients with Tourette syndrome. Mov Disord 2014;29:126-130.

41. Jankovic J. Therapeutic developments for tics and myoclonus. Mov Disord 2015;30: 1566-1573.
42. Jankovic J. Dopamine depletors in the treatment of hyperkinetic movement disorders. Expert Opin Pharmacother 2016;17:2461-2470.

43. Jankovic J, Jimenez-Shahed J, Budman C, et al. Deutetrabenazine in tics associated with Tourette syndrome. Tremor Other Hyperkinetic Mov 2016;6:422.

44. Zheng Y, Zhang ZJ, Han XM, et al. A proprietary herbal medicine (5-Ling Granule) for Tourette syndrome: a randomized controlled trial. J Child Psychol Psychiatry 2016;57:74-83.

45. Zhao L, Li AY, Lv H, Liu FY, Qi FH. Traditional Chinese medicine Ningdong granule: the beneficial effects in Tourette's disorder. J Int Med Res 2010;38:169-175.

46. Wang S, Qi F, Li J, Zhao L, Li A. Effects of Chinese herbal medicine Ningdong granule on regulating dopamine (DA)/serotonin (5-TH) and gamma-amino butyric acid (GABA) in patients with Tourette syndrome. Biosci Trends 2012;6:212-218.

47. Devinsky O, Cross JH, Laux L, et al; Cannabidiol in Dravet Syndrome Study Group. Trial of cannabidiol for drug-resistant seizures in the Dravet syndrome. New Engl J Med 2017;376:2011-2020.

48. Abrahamov A, Mechoulam R. An efficient new cannabinoid antiemetic in pediatric oncology. Life Sci 1995;56:2097-2102.

49. Elder JJ, Knoderer HM. Characterization of dronabinol usage in a pediatric oncology population. J Pediatr Pharmacol Ther 2015;20:462-467.

50. Kuhlen M, Hoell JI, Gagnon G, et al. Effective treatment of spasticity using dronabino in pediatric palliative care. Eur J Pediatr Neurol 2016;20:898-903.

51. Abi-Jaoude E, Chen L, Cheung P, Bhikram T, Sandor P. Preliminary evidence on cannabis effectiveness and tolerability for adults with Tourette syndrome. J Neuropsychiatry Clin Neurosci 2017;29:391-400.

52. Pilon P. Abide therapeutics reports positive topline data from phase $1 \mathrm{~b}$ study of ABX1431 in Tourette syndrome. 2017. Available at: abidetx.com. Accessed November 28 2017.

53. Martinez-Ramirez D, Jimenez-Shahed J, Leckman JF, et al. Efficacy and safety of deep brain stimulation in Tourette syndrome: the international Tourette syndrome deep brain stimulation public database and registry. JAMA Neurol 2018;75:353-359.

54. American Academy of Neurology. Clinical Practice Guideline Process Manual, 2011 St. Paul: The American Academy of Neurology; 2011.

\section{Now Accepting Applications for Emerging Leaders Program}

Applications are now open for the prestigious Emerging Leaders program, designed to identify, engage, and mentor talent among early-career members interested in future leadership roles within the American Academy of Neurology and the field of neurology. The application deadline is June 17. Learn more and apply at AAN.com/view/ELP.

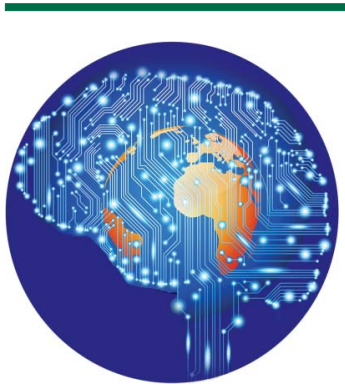

\section{Practice Current: An interactive exchange on controversial topics}

Share your own best practices.

Read commentary with expert opinion.

Explore results on an interactive world map.

NPub.org/NCP/practicecurrent

Neurology ${ }^{\circledR}$ Clinical Practice 


\section{Neurology}

Practice guideline recommendations summary: Treatment of tics in people with
Tourette syndrome and chronic tic disorders

Tamara Pringsheim, Michael S. Okun, Kirsten Müller-Vahl, et al.

Neurology 2019;92;896-906

DOI 10.1212/WNL.0000000000007466

This information is current as of May 6, 2019

\section{Updated Information \&} Services

References

Citations

Subspecialty Collections

Permissions \& Licensing

\section{Reprints}

including high resolution figures, can be found at: http://n.neurology.org/content/92/19/896.full

This article cites 49 articles, 2 of which you can access for free at: http://n.neurology.org/content/92/19/896.full\#ref-list-1

This article has been cited by 4 HighWire-hosted articles: http://n.neurology.org/content/92/19/896.full\#\#otherarticles

This article, along with others on similar topics, appears in the following collection(s):

\section{ADHD}

http://n.neurology.org/cgi/collection/adhd

Botulinum toxin

http://n.neurology.org/cgi/collection/botulinum_toxin

Tics

http://n.neurology.org/cgi/collection/tics

Tourette syndrome

http://n.neurology.org/cgi/collection/tourette_syndrome

Information about reproducing this article in parts (figures,tables) or in its entirety can be found online at:

http://www.neurology.org/about/about_the_journal\#permissions

Information about ordering reprints can be found online:

http://n.neurology.org/subscribers/advertise

Neurology ${ }^{\circledR}$ is the official journal of the American Academy of Neurology. Published continuously since 1951, it is now a weekly with 48 issues per year. Copyright () 2019 American Academy of Neurology. All rights reserved. Print ISSN: 0028-3878. Online ISSN: 1526-632X.

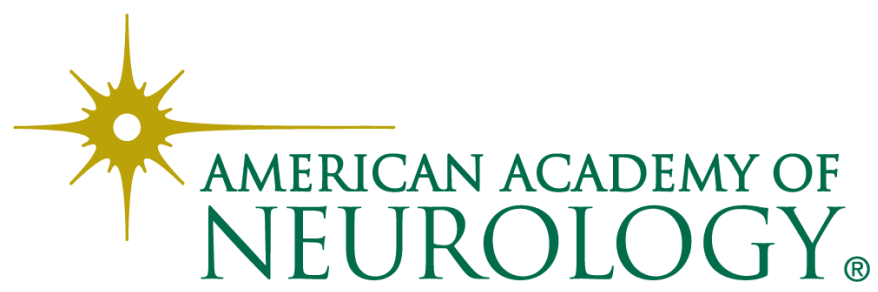

